\title{
D-dimer daily continuous tendency predicts the short-term prognosis for COVID-19 independently: A retrospective study from Northeast China
}

\author{
Yinting Xing, Wei Yang, Yingyu Jin, Chao Wang and Xiuru Guan* \\ Department of Clinical Laboratory, the First Affiliated Hospital of Harbin Medical University, \\ Harbin, Heilongjiang, China
}

\begin{abstract}
.
BACKGROUND AND OBJECTIVE: To study whether D-dimer daily continuous tendency could predict the short-term prognosis of COVID-19.

PATIENTS AND METHODES: According to the short-term prognosis, 81 COVID-19 patients were divided into two groups, one of worse prognosis (Group W) and the other of better prognosis (Group B). The slope of D-dimer linear regression during hospitalization (SLOPE) was calculated as an indicator of D-dimer daily continuous tendency. The SLOPE difference between Group W and Group B was compared. The difference between the discharge results and the 3-month follow-up results was also compared. COX regression analysis was used to analyze the relationship between SLOPE and short-term prognosis of COVID-19.

RESULTS: There were 16 patients in Group W and 65 patients in Group B. Group W had more critical proportion $(p<0.0001)$, indicating that the symptoms of its patients were more severe during hospitalization. ARDS, the most visible cause of worse prognosis, accounted for up to $68.75 \%$, and many symptoms merged and resulted in worse prognosis. The D-dimer levels of Group W not only were significantly higher $(p<0.0001)$, but also showed an increasing trend. In addition, the D-dimer levels at discharge were significantly higher than those at follow-up $(p=0.0261)$, and the mean difference was as high as 0.7474 . SLOPE significantly correlated with the short-term prognosis of COVID-19 independently (RR: 1.687, 95\%CI: 1.345-2.116, $P<0.0001)$. The worst prognosis occurred most likely during the first month after COVID-19 diagnosis.

CONCLUSION: Our study found that D-dimer daily continuous tendency independently correlates with worse prognosis and can be used as an independent predictor of the short-term prognosis for COVID-19.
\end{abstract}

Keywords: Coronavirus disease 2019 (COVID-19), SARS-CoV-2, D-dimer, prognosis, nomogram

\section{Background and objective}

Since December 2019, an outbreak of coronavirus disease 2019 (COVID-19) spread worldwide and the World Health Organization declared the COVID-19 as a pandemic on March 11, 2020 [1-3]. Upon viral infection of endothelial cells (ECs) by severe acute respiratory syndrome coronavirus 2 (SARS-CoV-2), ECs become activated and dysfunctional, which contribute to the elevated levels of proinflammatory cytokines, chemokines, von Willebrand factor antigen and its activity, and factor. Higher levels of acute phase reactants such as D-dimer are also associated with SARS-CoV-2 infection [4, 5].

\footnotetext{
*Corresponding author: Xiuru Guan, Department of Clinical Laboratory, the First Affiliated Hospital of Harbin Medical University, Nangang District, Harbin City, Heilongjiang Province, China. E-mail: guanxiuru0451@ 163.com.
} 
Therefore, it is reasonable to presume that endothelial dysfunction contributes to COVID-19-associated vascular inflammation, particularly endothelitis, in lung, heart, and kidney, as well as COVID-19associated coagulopathy, pulmonary fibrinous microthrombi in the alveolar capillaries [6, 7]. The endothelitis caused by the direct endothelial infection of SARS-CoV-2 and the indirect damage caused by inflammation play a remarkable role in the development of COVID-19-associated coagulopathy [8]. Therefore, we deduce that the tendency of D-dimer can predict the severity and outcome of COVID- 19. D-dimer daily continuous tendency could be calculated using the slope of linear regression depending on the daily D-dimer test results, which can be seen in the hospital information system. Our study aimed to study whether D-dimer daily continuous tendency could predict the short-term prognosis for COVID-19.

\section{Patients and methods}

\subsection{General information collection}

Retrospective analysis was performed on the clinical data of COVID-19 patients diagnosed at the COVID-19 Treatment Center in Heilongjiang Province from February to May 2020, including demographic and clinical diagnosis information. Diagnosis and classification of COVID-19 were based on China's COVID-19 Diagnosis and Treatment Protocol (Trial Version 7th, promulgated by the National Health Commission of China and the National Administration of Traditional Chinese Medicine, no English version). There are four types of COVID-19: light, moderate, severe, and critical. Basic diseases include respiratory failure, hypertension, thrombotic diseases (upper and lower limb vein, portal vein thrombosis), diabetes mellitus, coronary heart disease (CHD), liver injury, anemia, infarction (lacunar cerebral infarction, old cerebral infarction), hypoproteinemia, atrial fibrillation (AF), etc. Patients with COVID-19 were followed up for 3 months to record their short-term prognosis. During the follow-up, the worse prognosis was defined as coma, death and other malignant events, and the time as well as the clinical diagnosis were recorded when the worse prognosis occurred. The better prognosis was defined as the cure of COVID-19 without observing malignant events such as coma and death. According to the short-term prognosis, COVID-19 patients were divided into one group of worse prognosis (Group W) and another group of better prognosis (Group B). The identity documents of patients in the two groups were IDW and IDB respectively. Statistics show that a total of 130 COVID-19 patients were diagnosed and treated in our center, including 24 patients whose D-dimer levels were not tested and 25 patients whose D dimer levels were not tested continuously (defined as having 3 or more). Therefore, 81 COVID-19 patients were included in this study (Fig. 1).

\subsection{D-dimer sample collection and detection}

D-dimer samples were collected from the peripheral venous blood of COVID-19 patients with $109 \mathrm{mmol} / \mathrm{L}$ sodium citrate anticoagulant tube ( $3 \mathrm{ml}$, BD Company, USA). A low-speed centrifuge was used to obtain plasma at $3500 \mathrm{rpm}$ for $10 \mathrm{~min}$. The detection reagent was an original D-dimer detection kit produced by Japan SEKISUI Company, and the detection instrument was a Coapresta 3000 of Japan SEKISUI automatic coagulation analyzer. The unit was DDU: $\mu \mathrm{g} / \mathrm{ml}$, and the cutoff value was 1.0 DDU $(\mu \mathrm{g} / \mathrm{ml})$. The D-dimer levels of COVID-19 patients were tested consecutively during hospitalization and once at 3-month follow-up. The test frequency was $0.5-4$ times per day based on the needs of patients during hospitalization. The average of D-dimer levels tested multiple times on the same day was used as the test result of D-dimer on the same day. The slope of linear regression of D-dimer results (SLOPE) during hospitalization of each COVID-19 patient was calculated 

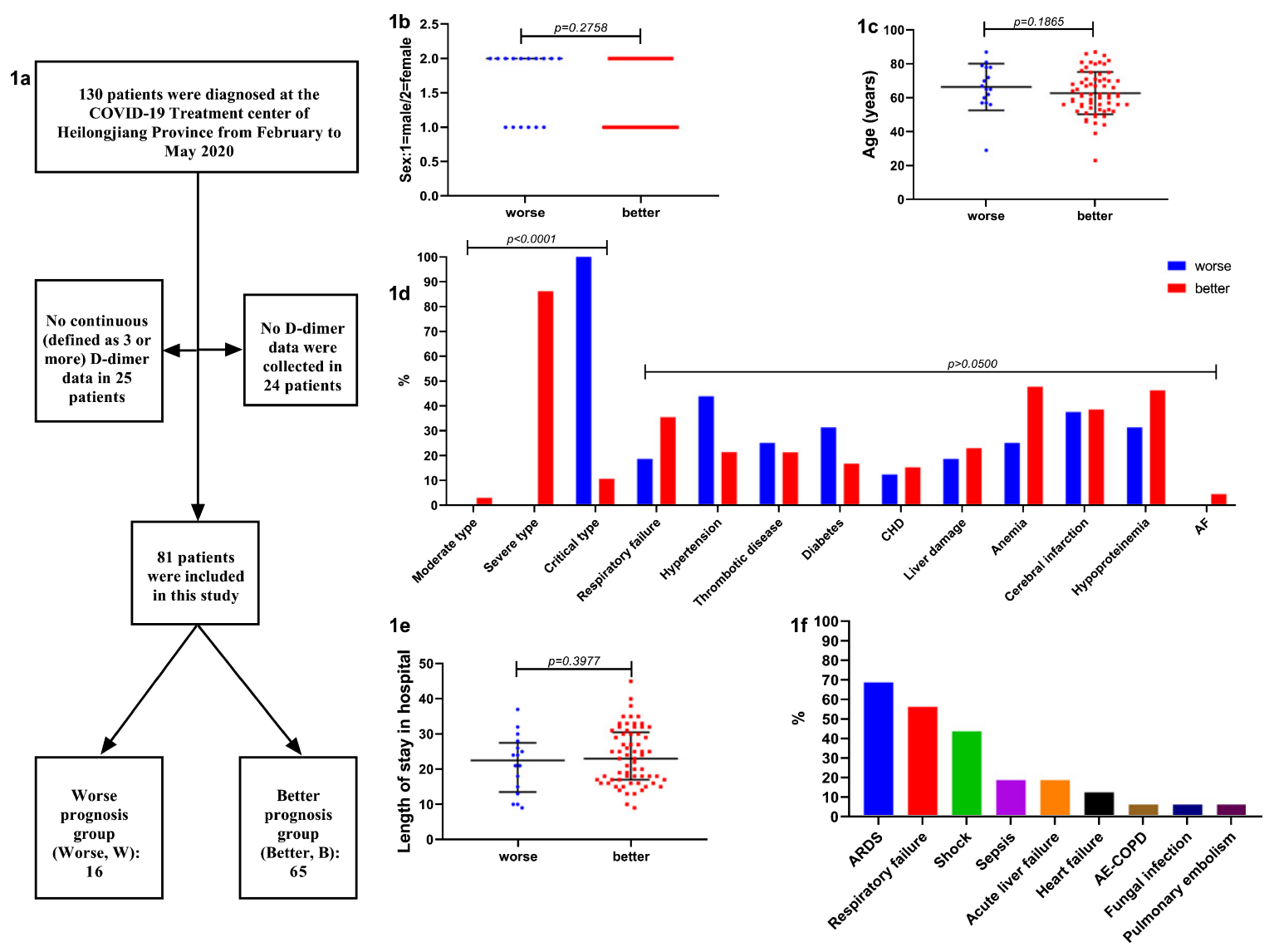

Fig. 1. a: The flowchart of subjects included in this study. b: Sex differences between the two groups was not significant. c: Age differences between the two groups was not significant. d: COVID-19 types differences between the two groups was significant $(p<0.0001)$, all COVID-19 type in Group W was critical, while the proportion of each type in Group B was moderate $(3.1 \%)$, severe $(86.2 \%)$ critical $(10.8 \%)$, respectively. But the basic disease composition between the two groups was not significant. e: Length of stay in hospital differences between the two groups was not significant. f: When the worse prognosis was occurred, clinical diagnosis was analyzed, visible causes of worse prognosis were ARDS, respiratory failure (type I), shock (including septic shock and cardiac shock), sepsis, acute liver failure, heart failure, AE-COPD, fungal infection, pulmonary embolism. ARDS accounted for the up to 68.75\%. Abbreviations: CHD: coronary heart disease; AF: atrial fibrillation; ARDS: acute respiratory distress syndrome; AE-COPD: acute exacerbation of chronic obstructive pulmonary disease.

as an indicator of D-dimer daily continuous tendency, and the difference in SLOPE between Group W and Group B was compared. D-dimer levels were tested once during the follow-up as the control result. The difference between the discharge results and the follow-up results of Group B patients was compared, and the change pattern of D-dimer levels during COVID infection was analyzed.

\subsection{Statistical analysis}

The measurement data was expressed as mean $\pm \mathrm{SD}$ or median (P25, P75) interquartile spacing, and the counting data was expressed as percentage. T-test was used for the comparison of normal distribution measurement data; non-parametric test was used for the comparison of non-normal distribution measurement data, counting data and grade data (Mann-Whitney U test). Spearman correlation analysis was used for univariate and multivariate correlation analysis; COX regression was used for univariate 
and multivariate regression analysis; and $p$-value $<0.05$ was used for all items included in regression equation. COX regression analysis was used to analyze the factors affecting the worse prognosis of COVID-19 patients, and to analyze whether SLOPE could be used as a predictor. SPSS19.0 statistical Software (IBM Inc., USA) was used for data analysis; GraphPad Prism 7.0 Software (GraphPad Software Inc., USA) was used for graphics rendering; and COVID-19 survival factor COX nomogram was drawn by RStudio (RStudio, Inc., USA), $\alpha=0.05$.

\subsection{Ethical issues}

The Ethics Committee of the First Affiliated Hospital of Harbin Medical University approved this study (No. 202014), and all enrolled patients gave their written informed consent.

\section{Results}

\subsection{General data analysis}

In this study, 81 COVID-19 patients, 16 in Group W and 65 in Group B, were successfully included (Fig. 1a). We analyzed the sex, age, COVID-19 type, and length of stay between the two groups. One statistically significant difference between the two groups was the COVID-19 type $(p<0.0001)$ : the COVID-19 type of all patients in Group W was critical, while the proportion of each type in Group B was moderate $(3.1 \%)$, severe $(86.2 \%)$, and critical $(10.8 \%)$ respectively, showing that Group W had more critical patients than Group B, and the symptoms were more severe during hospitalization (Fig. 1b-1e). There was no significant difference in the basic symptom composition between the two groups. When the worse prognosis occurred, the clinical diagnosis was analyzed, showing visible causes of worse prognosis were acute respiratory distress syndrome (ARDS), respiratory failure (type I), shock (including septic shock and cardiac shock), sepsis, acute liver failure, heart failure, acute exacerbation of chronic obstructive pulmonary disease (AE-COPD), fungal infection, and pulmonary embolism. ARDS accounted for the largest proportion, up to $68.75 \%$, and many diseases often appeared to merge and resulted in worse prognosis, the worst even exhibiting 6 different diseases at the same time (Fig. 1f).

\subsection{Analysis of D-dimer results showed that SLOPE positively correlated with poor prognosis of COVID-19}

Through analysis, we found that not only the D-dimer levels in Group W were significantly higher than Group B ( $p<0.0001)$ (Fig. 3c), but the test results showed an increasing trend as well (Figs. 2, 3a$3 b)$. The slope of the D-dimer level trend was calculated by linear regression, and a significant difference was also found between the two groups $(p=0.0022)$ (Fig. 3d). Correlation analysis showed that SLOPE significantly positively correlated with short-term worse prognosis $(p=0.0381)$. In addition, $\mathrm{D}$-dimer SLOPE significantly correlated with age $(p=0.0027)$ and diabetes diagnosis $(p=0.0140)$. Furthermore, we analyzed the difference in D-dimer levels between discharge and follow-up. D-dimer levels at discharge were significantly higher than those at follow-up $(p=0.0261)$, with mean difference as high as 0.7474 (Fig. 3e). 

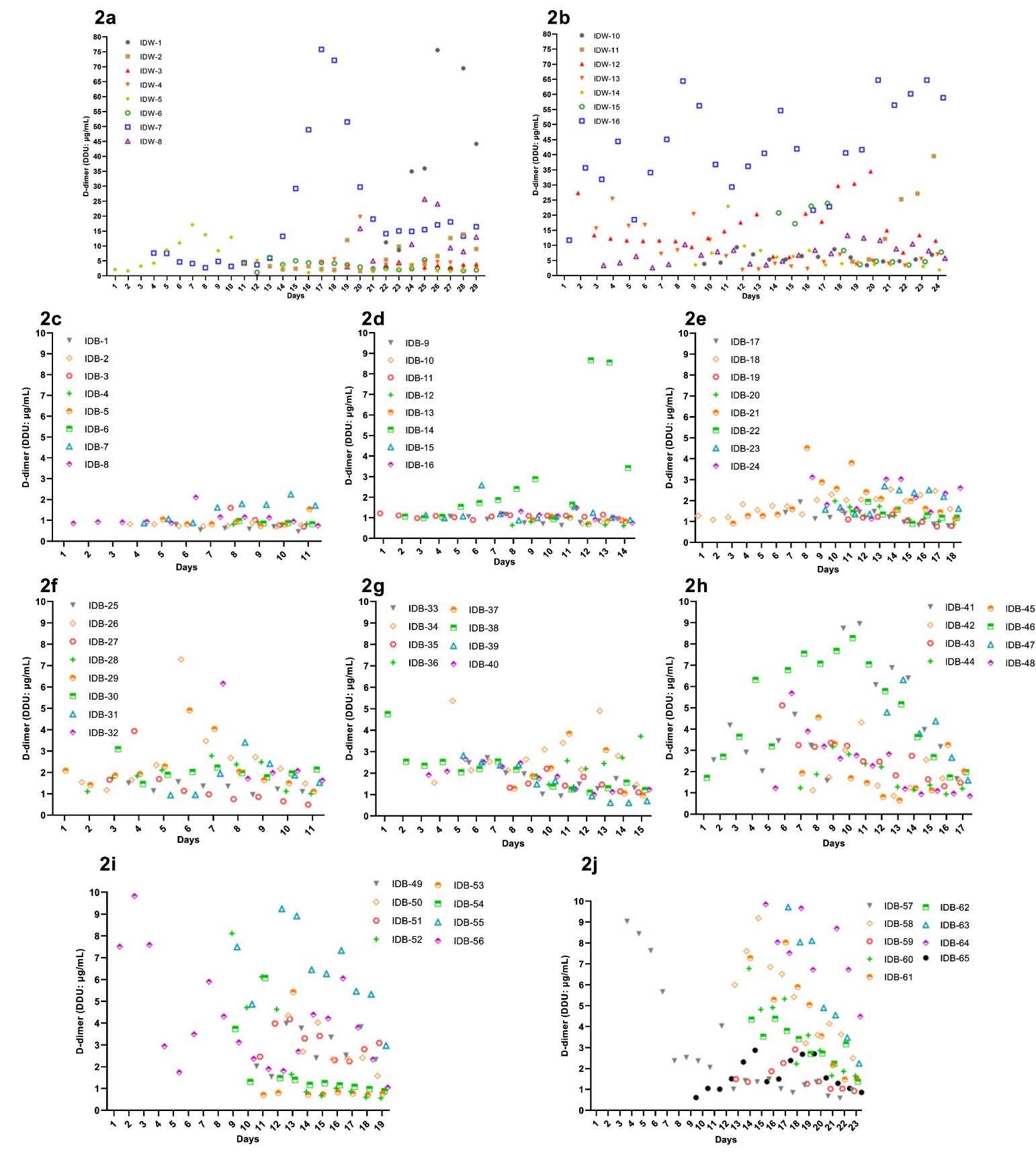

Fig. 2. D-dimer detection results of every patient in the two groups.

\subsection{COX regression analysis showed that SLOPE was an independent predictor for short-term prognosis of COVID-19}

Univariate COX regression analysis showed that SLOPE significantly positively correlated with short-term worse prognosis of COVID-19 (RR: 1.414, 95\%CI: 1.186-1.678, $p=0.0001$ ). Meanwhile, ARDS (RR: 21.202, 95\%CI: 7.369-61.002, $p<0.0001$ ), shock (RR: 19.184, 95\%CI: 6.799-54.132, $p<0.0001$ ), sepsis (RR: 15.016, 95\%CI: 3.986-56.830, $p<0.0001)$ and heart failure (RR: 6.892, 

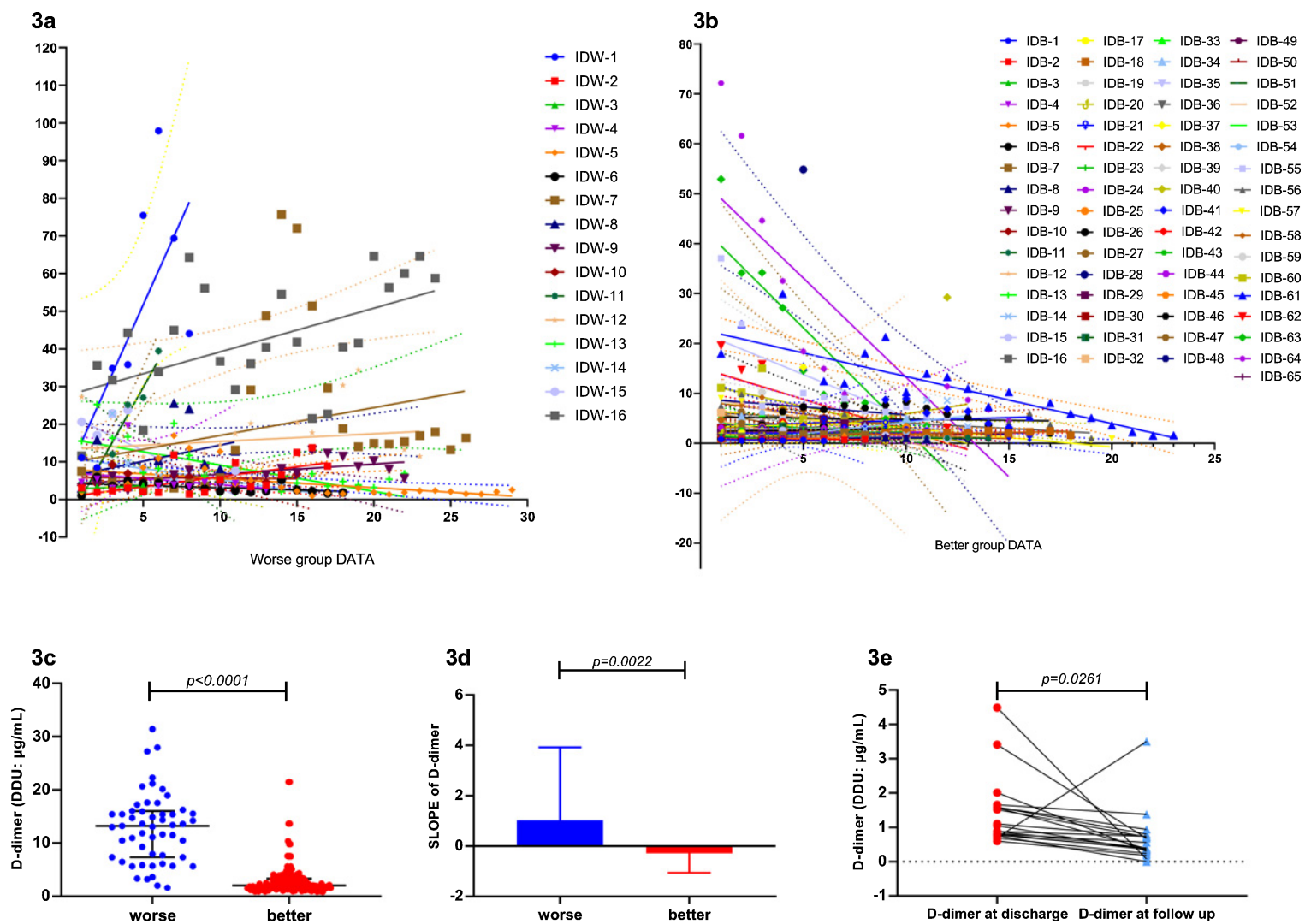

Fig. 3. 3a. D-dimer trends of Group W were increased. 3b. D-dimer trends of Group B were decreased. 3c. D-dimer detection results in Group W were significantly higher than Group B $(p<0.0001)$. 3d. The SLOPE of the Ddimer trend in Group W were significantly higher than Group B $(p=0.0022)$. D-dimer levels at discharge were significantly higher than that at follow up $(p=0.0261)$.

95\%CI: $1.542-30.814, p=0.0115)$ were found to significantly correlate with short-term worse prognosis of COVID-19. When conducting multivariate COX regression analysis, we excluded heart failure due to insufficient data. Therefore, we incorporated the first 4 results and conducted multivariate COX regression analysis. We found that SLOPE still significantly correlated with the short-term prognosis of COVID-19 (RR: 1.687, 95\%CI: 1.345-2.116, $p<0.0001$ ). Finally, nomogram was drawn for the short-term prognosis of COVID-19, and the results were further presented in Fig. 4. The nomogram in our study also showed that the worst prognosis occurred most likely during the first month after COVID-19 was diagnosed.

\section{Discussion}

COVID-19 has been the pandemic around the world by its highly sensitive to every single person and has led to a widespread mortality [9]. Studies focused on the risk factors and predictors for the prognosis of COVID-19 have been conducted for months since COVID-19 was first discovered [10-13]. Despite several studies on the correlation between D-dimer levels and prognosis of COVID-19, the study on the relationship between D-dimer daily continuous tendency and the 3 months' prognosis is rarely seen. Our study found that the D-dimer variation degree independently correlated with worse prognosis, and could be used as an independent predictor of short-term prognosis for COVID-19. Monitoring 

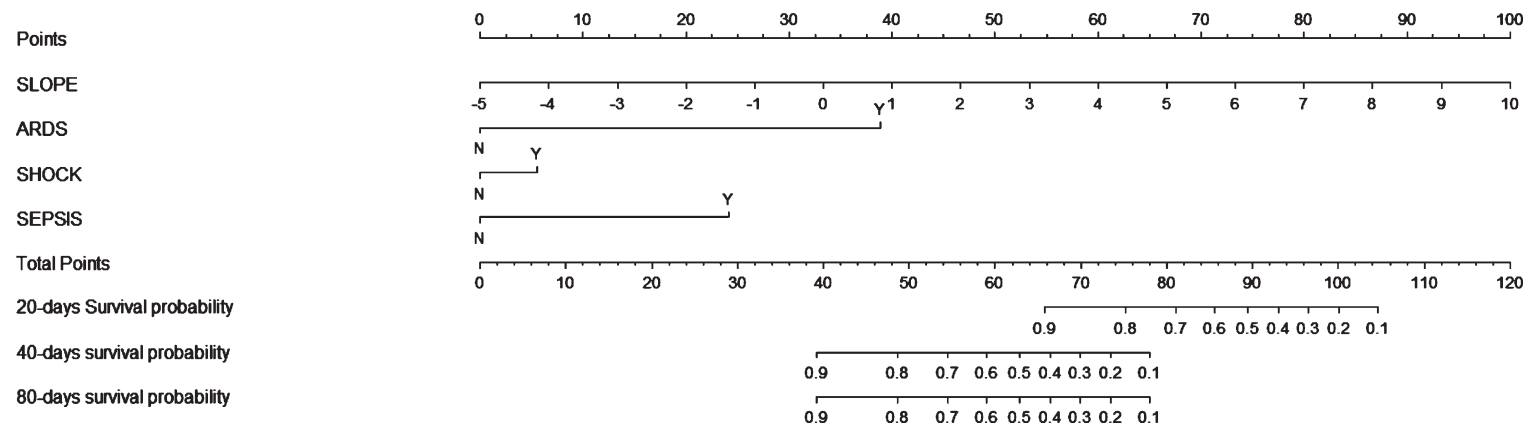

Fig. 4. Nomogram was drawn for the short-term prognosis of COVID-19, showed that the worst prognosis occurred most likely during the first month after COVID-19 diagnosis.

this indicator during the hospitalization can play an important role in predicting the prognosis of COVID-19 patients.

Nomograms of D-dimer level were studied to predict the prognosis of COVID-19 patients [14, 15], which showed that D-dimer levels were associated with mortality of COVID-19 patients. Several metaanalyses also showed that a significantly increased D-dimer had a malign impact on the mortality among COVID-19 patients [16-18]. The presence of elevation of D-dimer level ( $>6$ times the normal value) was associated with worse prognosis in COVID-19 patients during hospitalization, with a hospital mortality rate of $42 \%$ [1]. In our study, the average D-dimer level in Group W was also significantly higher than that in Group B, exhibiting the same result as in previous studies. The nomogram in our study also showed that the worst prognosis occurred most likely during the first month after COVID-19 diagnosis.

D-dimer daily continuous tendency in our study independently correlated with worse prognosis. The angiotensin converting enzyme 2 receptor (ACE2 receptor) is expressed on ECs, which mainly in heart, lung, kidney, intestine, and particularly in systemic vessels (arteries, veins, venules, and capillaries) [6]. Thus, in our study, we found that many diseases often appeared to merge and resulted in worse prognosis, the worst even exhibiting 6 diseases at the same time. Cell entry is initiated by the recognition of the host cell ACE2 receptor by the receptor-binding domain (RBD) of the spike protein of SARS-CoV-2 [19]. The occurrence of specific comorbidities associated with renin-angiotensin system (RAS) imbalance is mediated by the interaction between ACE2 and metalloproteinase domain 17 (ADAM17), along with specific genetic factors mainly associated with type II transmembrane serine protease (TMPRSS2) expression. Indeed, the exacerbated ADAM17 mediated ACE2, TNF- $\alpha$, and IL6R secretion emerges as a possible underlying mechanism for the acute inflammatory immune response and the activation of the coagulation cascade [20]. Thus, the elevated D-dimer levels might be caused by the following reasons. First, severe viral induced cytokine storms activated coagulation cascade. Pro-inflammatory cytokines stimulated the expression of tissue factors on immune cells and then activated trigger exogenous coagulation cascade. Second, the activity of urokinase-type plasminogen activator was reduced, and the release of plasminogen activator inhibitor- 1 was elevated, thus inhibiting the fibrinolysis system. Third, platelets were activated by various pro-inflammatory cytokines and combined with damaged endothelium. Fourth, endothelial injury caused by inflammation accelerated the thrombosis response [21]. A retrospective analysis showed that in COVID-19 patients, D-dimer levels were related to markers of inflammation but not venous thromboembolism (VTE) score [22]. In our study, patients in Group W were in such critical condition that most of them had to lie in bed during hospitalization, and some of them received endotracheal intubation, mechanical ventilation or even Extracorporeal Membrane Oxygenation (ECMO) in the last few days to alleviate respiratory disorders. 
For these reasons the elevation of D-dimer daily continuous tendency in our study might be caused by both COVID-19 infection and thromboembolism.

A reporting of D-dimer in COVID-19 concerned that potentially under-recognized by those reporting on D-dimer is the considerable variation in reporting mode, thus the potential for misreporting of Ddimer based on poor or incomplete reporting [23]. In our study, we provided information including the unit (DDU: $\mu \mathrm{g} / \mathrm{ml}$ ), cutoff value, reagent, machine, so that our study can be clearly comparable with other studies.

\section{Conclusion}

Our study found that D-dimer daily continuous tendency independently correlates with worse prognosis and can be used as an independent predictor of short-term prognosis for COVID-19. The worst prognosis occurred most likely during the first month after COVID-19 was diagnosed. Visible causes of worse prognosis include acute respiratory distress syndrome (ARDS), respiratory failure (type I), shock (including septic shock and cardiac shock), sepsis, acute liver failure, heart failure, acute exacerbation of chronic obstructive pulmonary disease (AE-COPD), fungal infection, and pulmonary embolism. ARDS accounted for up to $68.75 \%$, and many diseases often appeared to merge and resulted in worse prognosis. These observations might be helpful in clinical practice for COVID-19.

\section{Conflicts of interest}

The authors declare no conflict of interest.

\section{Funding}

This research received Research and Innovation Fund of the First Affiliated Hospital of Harbin Medical-Technical research project for the prevention and treatment of COVID-19. Fund No. 2020 YJ03.

\section{References}

[1] Gómez-Mesa JE, Galindo-Coral S, Montes MC, Muñoz Martin AJ. Thrombosis and Coagulopathy in COVID-19. Curr Probl Cardiol. 2020:100742.

[2] Chang WH. The influences of the COVID-19 pandemic on medical service behaviors. Taiwan J Obstet Gynecol. 2020;59(6):821-7.

[3] Jung E, Stroszczynski C, Jung F. Contrast enhanced ultrasound (CEUS) to assess pleural pulmonal changes in severe COVID-19 infection: First results. Clin Hemorheol Microcirc. 2020;75(1):19-26.

[4] Rostami M, Mansouritorghabeh H. D-dimer level in COVID-19 infection: a systematic review. Expert Rev Hematol. 2020:1-11.

[5] Zhang L, Yan X, Fan Q, Liu H, Liu X, Liu Z, et al. D-dimer levels on admission to predict in-hospital mortality in patients with Covid-19. J Thromb Haemost. 2020;18(6):1324-9.

[6] Zhang J, Tecson KM, McCullough PA. Endothelial dysfunction contributes to COVID-19-associated vascular inflammation and coagulopathy. Rev Cardiovasc Med. 2020;21(3):315-9.

[7] Jung F, Krüger-Genge A, Franke RP, Hufert F, Küpper JH. COVID-19 and the endothelium. Clin Hemorheol Microcirc. 2020;75(1):7-11.

[8] Iba T, Connors JM, Levy JH. The coagulopathy, endotheliopathy, and vasculitis of COVID-19. Inflamm Res. 2020;69(12):1181-9. 
[9] Veldtman GR, Pirisi M, Storti E, Roomi A, Fadl-Elmula FEM, Vriz O, et al. Management principles in patients with COVID-19: perspectives from a growing global experience with emphasis on cardiovascular surveillance. Open Heart. 2020;7(2).

[10] Figliozzi S, Masci PG, Ahmadi N, Tondi L, Koutli E, Aimo A, et al. Predictors of adverse prognosis in COVID-19: A systematic review and meta-analysis. Eur J Clin Invest. 2020;50(10):e13362.

[11] Mudatsir M, Fajar JK, Wulandari L, Soegiarto G, Ilmawan M, Purnamasari Y, et al. Predictors of COVID-19 severity: a systematic review and meta-analysis. F1000Res. 2020;9:1107.

[12] Li K, Chen D, Chen S, Feng Y, Chang C, Wang Z, et al. Predictors of fatality including radiographic findings in adults with COVID-19. Respir Res. 2020;21(1):146.

[13] Alzoughool F, Alanagreh L, Abumweis S, Atoum M. Cerebrovascular comorbidity, high blood levels of C - reactive protein and D-dimer are associated with disease outcomes in COVID-19 patients. Clin Hemorheol Microcirc. 2020. doi: 10.3233/CH-201002

[14] Cheng A, Hu L, Wang Y, Huang L, Zhao L, Zhang C, et al. Diagnostic performance of initial blood urea nitrogen combined with D-dimer levels for predicting in-hospital mortality in COVID-19 patients. Int J Antimicrob Agents. 2020;56(3):106110.

[15] Ma X, Wang H, Huang J, Geng Y, Jiang S, Zhou Q, et al. A nomogramic model based on clinical and laboratory parameters at admission for predicting the survival of COVID-19 patients. BMC Infect Dis. 2020;20(1):899.

[16] Yang L, Jin J, Luo W, Gan Y, Chen B, Li W. Risk factors for predicting mortality of COVID-19 patients: A systematic review and meta-analysis. PLoS One. 2020;15(11):e0243124.

[17] Zhou X, Cheng Z, Shu D, Lin W, Ming Z, Chen W, et al. Characteristics of mortal COVID-19 cases compared to the survivors. Aging (Albany NY). 2020;12.

[18] Kiss S, Gede N, Hegyi P, Németh D, Földi M, Dembrovszky F, et al. Early changes in laboratory parameters are predictors of mortality and ICU admission in patients with COVID-19: a systematic review and meta-analysis. Med Microbiol Immunol. 2020:1-15.

[19] Bhattacharjee S. Toward COVID-19 Therapeutics: A Viewpoint from the Nonprotein Amino Acid Based Synthetic Peptide Design Approach. ACS Chem Neurosci. 2020;11(22):3701-3.

[20] Zipeto D, Palmeira JDF, Argañaraz GA, Argañaraz ER. ACE2/ADAM17/TMPRSS2 Interplay May Be the Main Risk Factor for COVID-19. Front Immunol. 2020;11:576745.

[21] Hadid T, Kafri Z, Al-Katib A. Coagulation and anticoagulation in COVID-19. Blood Rev. 2020:100761.

[22] Yu B, Li X, Chen J, Ouyang M, Zhang H, Zhao X, et al. Evaluation of variation in D-dimer levels among COVID-19 and bacterial pneumonia: a retrospective analysis. J Thromb Thrombolysis. 2020;50(3):548-57.

[23] Favaloro EJ, Thachil J. Reporting of D-dimer data in COVID-19: some confusion and potential for misinformation. Clin Chem Lab Med. 2020;58(8):1191-9. 